\title{
The Impact of Surface and Geometry on Friction Coefficient Behavior of Artificial Hip Joints
}

Dipankar Choudhury ${ }^{1,2 \#}$, Martin Vrbka ${ }^{2,3}$, Azuddin Bin Mamat ${ }^{4}$, Ian Stavness ${ }^{1}$, Chanchal K. Roy $^{1}$, Rajshree Mootanah ${ }^{5}$, Ivan Krupka ${ }^{2,3}$

${ }^{1}$ Department of Computer Science, University of Saskatchewan, 176 Thorvaldson Building, 110 Science Place, Saskatoon, SK, S7N 5C9 Canada

${ }^{2}$ Faculty of Mechanical Engineering, Brno University of Technology, Technicka 2896/2, 61669 Brno, Czech Republic

${ }^{2}$ CEITEC-Central European Institute of Technology, Brno University of Technology, Technicka 3058/10, 61600 Brno, Czech Republic

${ }^{3}$ Institute of Machine and Industrial Design, NETME Centre, Brno University of Technology, Technicka 2896/2, 61669 Brno, Czech Republic.

${ }^{4}$ Department of Mechanical Engineering, Faculty of Engineering, University of Malaya, 50603 Kuala Lumpur, Malaysia

${ }^{5}$ Faculty of Science and Technology, Anglia Ruskin University, Chelmsford, Essex CM1 1SQ, UK

\#corresponding authors: dipankar.choudhury78@gmail.com, TEL: +13069664892, FAX: +001 966-4884

\begin{abstract}
:
Friction coefficient tests were conducted on 28-mm and 36-mm-diameter (Ø) hip joint prostheses of the four different material combinations, with or without the presence of Ultra High Weight Molecules Polyethylene (UHWMPE) particles using a novel pendulum hip simulator. The effects of three micro dimpled arrays on prosthesis head against a polyethylene cup and a metallic cup were investigated. Clearance played a vital role in friction coefficient of various geometrical and materials oriented artificial hip joints. Micro dimpled metallic heads yielded higher friction coefficient against polyethylene cup, however, these dimpled arrays were significantly effective in friction coefficient reducing to metal on metal prosthesis. The in situ images disclose the evidence of enhanced film formation due to the dimple, a main mechanism that contributed to reduced friction.
\end{abstract}

Keywords: Hip joint prosthesis, geometry of prosthesis, micro dimple, friction coefficient, lubrication film thickness 


\section{Introduction:}

The number of hip replacement procedures is more than 2 million per annum worldwide, which will increase twofold by 2020 because of increasing aging population ${ }^{1,2}$. According to the 2014 Canadian Joint Replacement Registry, the numbers of hip and knee replacements have increased by $16.5 \%$ and $21.5 \%$, respectively, over the last five years ${ }^{3}$. The demographics include an increasing number of younger patients (45-64 years) and thus, prostheses are now required to last over 30 years $^{3-5}$.

The orthopedics device manufacturing companies, equipped with advanced manufacturing facilities, are able to produce prostheses with high precision and very smooth surface (10-20 $\mathrm{nm})$. Although ISO standard is followed during in vitro testing, revision rates of prostheses in clinical application are as high as 8-10\%, within an average 15-year lifespan per device, according to the UK and Canadian national survey reports ${ }^{3,4}$. Surgical techniques and precision, as well as patient-related physiological factors and activity levels are important determining factors for the survival rates of implanted hip or knee joints ${ }^{6}$. In addition to these factors, excessive wear rate and its associated debris are the major drivers of revision surgery ${ }^{7,8}$. Wear is a complex mechanism, involving lubrication and coefficient of friction (REF- Pucciobiotribology). Scholes et al, showed that lower friction factors are associated with thicker lubrication film formation. It is noted that articular cartilage along with synovial fluid, play the key role in protecting the joint interface from mechanical wear and facilitating a smooth motion (friction coefficients can reach as low as 0.001) [30, 31]. Thus Friction Coefficient (FC), wear rate and their associated debris are the main measurable outcomes to evaluate the performance of newly developed hip prostheses. 
One of the widely-used methods of determining FC is based on strain gauge, principally suitable to pin-on-disk tribo-tester (non-conformal contact) ${ }^{9}$. A hip joint is a ball-and-socket joint with conformal contact. Hence, it is challenging to measure FC and real-time wear rate, using a strain gauge. Thus, the measurable outcomes for determining FC, using hip simulators, are generally focused on the evaluation of wear rate only ${ }^{10,11}$. The pendulum hip simulator is a novel device $^{10}$ that can be used to obtain real-time velocity profile and estimated frictional coefficient data of hip prosthesis, while accurately maintaining prosthesis geometrical configurations, such clearance and sizes.

Prosthesis size and clearance are important selection criteria for hip replacement ${ }^{12,13}$. A 28 mm-diameter (Ø) hip prosthesis is widely used in many countries ${ }^{14}$. Despite of previous studies $^{13,15}$ have shown that hip joint prostheses with a larger diameter have lower wear rate, their fundamental tribological behavior and lubrication viscous effect are not yet clearly understood. Similarly, no single study has investigated the effect of size and clearance on FC with all of the major material pairs, such as metal-on-metal (MoM), ceramic-on-ceramic (CoC), metal-on-polyethylene (MoP) and ceramic-on-polyethylene (CoP) prostheses. Similarly, in the current literature, Only a few articles mentioned the effect of wear debris in third body abrasive wear mechanism and a possible reduction by surface modification ${ }^{16,17}$. Micro-dimpling is a method of surface modification used in many engineering applications, such as in engine cylinders $^{18,19}$, ball and rolling bearings $s^{20,21}$ and has potential in orthopedics ${ }^{22,23}$. Micro-dimpling can increase hydrodynamic pressure by enhancing film formation; it acts a reservoir to, simultaneously, increase lubrication and entrap debris. 
However, a synovial-lubricated micro dimpled-conformal contact (i.e. the bearing in hip and knee joints with defined size and clearance) is not yet fully understood. Eleven articles have been published investigating the effect of surface texturing on orthopedic implants; six considered 'MoP' 16,17,23-26, one ' $\mathrm{CoC}$ '22, and the remaining four, 'MoM' implants ${ }^{27-30}$; Four patents have been granted based on these endeavors ${ }^{31-34}$. The dimple parameters utilized were diverse, but most of them demonstrated that a micro-dimpled surface reduced friction and wear. Among the MoM prosthesis, two are theoretical and other two are based honed surfaces. Therefore, a micro dimple surface technique is yet to be investigated in metal-on-metal hip joints.

In this study, four types of FC experiments were conducted, including a) a 28-mm $\varnothing$ and a 36-mm Ø prosthesis with four material combinations (MoM, MoP, CoC and CoP); b) the impact of polyethylene particles in the MoP hip joints interface; and c) the effect of three micro dimple arrays on MoP and MoM prostheses.

\section{Material and Methods}

2.1. Materials: Hip joint prostheses with diameters of $28-\mathrm{mm}$ and $36-\mathrm{mm} \varnothing$ and in four material combinations, including MoM, MoP, $\mathrm{CoC}$ and $\mathrm{CoP}$, as detailed in Table 1, were obtained from University Hospital Olomouc, Czech Republic. The 36- mm $\varnothing$ MoM had the largest clearance $(609 \mu \mathrm{m})$ and the $28 \mathrm{~mm} \varnothing \mathrm{MoM}$ has lowest clearance $(274 \mu \mathrm{m})$. Clearance and contact surfaces were measured, using a 3D optical scanner active fringe projection (GOM ATOS Triple Scan, GOM mbH, Germany). The accuracy of the measuring system is justified according to the acceptance test (VDI/VDE 2634 - Part 3) whereas the probing error size is $\pm 0.005 \mathrm{~mm}$. The Post-processing calculate on the diameter of the spherical surface of prosthesis was performed, using the ATOS professional software. The exact diameter of contact surface of 
artificial glass cups were 28.08 and $38.08 \mathrm{~mm}$. Artificial femoral heads (details in the table 1) were delivered in the original package from the manufacturer and the exact diameter after scanning was 27.988 and $35.299 \mathrm{~mm}$.

UHMWPE powder ET306010 (Goodfellow Cambridge Ltd, Huntingdon, UK) was used to test the influence of debris on wear rate. To identify a reliable circumference value, we measured 5 random particles, 3 times each. The particles were first sputtered on the microscope slide and placed into the profile-meter. Three images were captured from different slide orientations and analyzed, using a 3D optical microscopy (Contour GT-I, Brucker, Italy). The edges in all the images were identified and broken grains were discarded. The particles supplied had an average equivalent circumference of $150 \mu \mathrm{m}$, but the deviation was large, $100 \mu \mathrm{m}$.

Three types of micro dimples arrays (square, triangular and circular) were fabricated on the $28 \varnothing$ Aesculap, Isodur (metal) prosthesis heads. Indentation techniques ${ }^{21}$ were used to produce the square and triangle arrays, and micro drilling was performed to create circular dimple arrays. A 3-axis rotation axis device was used to produce dimples on the prostheses because of their spherical shape. The indentation has one axis of rotation ( $Z$ ) and two axis planner movement facilities, which allowed the fabrication of the square and triangular micro dimple arrays on the top 6x6 $\mathrm{mm}^{2}$ area of the prosthesis. The outer ring dimples were unavoidably slightly tilted from the perpendicular position. The tip of the indenter was tilted at an angle of $15^{\circ}$ from the vertical axis. The CNC micro drilling machine, with three rotational axes, enabled fabrication of dimples perpendicularly to the prosthesis head. The tungsten carbide WC drill bit of $200 \mu \mathrm{m} \varnothing$ was used to produce the circular dimple arrays. The dimple parameters are presented in Table 2, and the 3D images are shown in Fig. 1. The images and their roughness 
profile were captured and evaluated, using a 3D optical microscopy (Contour GT-I, Brucker, Italy). The images showed presence of protuberance formations around the dimples, which were removed by a further surface finishing, using 0.5 micron diamond paste. The dimple roughness profiles before and after the polishing are shown in Fig. 2.

\subsection{Friction test}

Tribology tests were performed, using a novel pendulum hip joint simulator (Fig. 3), described by Choudhury et $\mathrm{al}^{35,36}$. The experimental parameters are shown in Table 3.

The prosthesis head is loaded by two handed weight bar which can be loaded and unloaded manually. The pendulum is pulled up to $16^{\circ}$, and then released to swing freely. The frequency and the initial peak amplitude were $0.5 \mathrm{~Hz}$ and 0.9 radians, respectively. The amplitude reduced to zero over time due to the FC. The measured angular velocity profile was transferred to the computer and FC was calculated from the recorded values of angular amplitudes. The applied load was $2 \mathrm{KN}$, simulating three times body weight during a standard walking gait for a MoC hip joint ${ }^{37,38}$. Bovine serum (25\%) was used as lubricant, and temperature was maintained at $37^{\circ} \mathrm{C}$ to simulate body temperature.

\subsection{Computational simulation:}

A numerical analysis (ANSYS R.15) was carried out to investigate the deformation of softer UHWMPE against dimpled metallic head. The numerical model consisted of two planar bodies. The dimpled surface was set on the bottom with fixed mode and non-dimpled cup was loaded with contact pressures of $10 \mathrm{MPa}$ and $24 \mathrm{MPa}$. The dimple was $250 \mu \mathrm{m}$ in diameter and $40 \mu \mathrm{m}$ in depth, which corresponded to the dimple geometry of the circular array. 


\section{Result and Analysis}

\subsection{Effect of Prosthesis Size on Friction Coefficient:}

The effect of prosthesis size and clearance on FC of MoP and CoP prostheses are shown Fig. 4. - FC of the 36-mm-Ø MoP prosthesis started from 0.12, increased gradually, and finally settled to 0.18 after ten test repetitions in steps of 3 minutes. FC of the $28-\mathrm{mm}-\varnothing$ MoP prosthesis started from 0.14 and gradually increased to 0.19 after ten repetitions. Therefore, the FC trends of the MoP hip prostheses of different sizes were very similar. MoP prosthesis size did not have noticeable influence on FC. FC of the 36-Ø-CoP prosthesis started from 0.095 for the first experiment and extended to 0.11 at its $10^{\text {th }}$ repetition, which was lower than that of $28-\mathrm{mm}-\varnothing$ CoP (13\% lower at the first experiment and $22 \%$ after the $10^{\text {th }}$ repetition). Overall, CoP FCs are much lower compared to MoP FCs, regardless of their sizes.

MoM and MoP prostheses had similar FC trends. FC was not influenced by the prostheses of different sizes (28-mm and 36-mm Ø). However, the maximum FC of MoM (0.16) was lower than that of MoP (0.19). On the other hand, CoC exhibited a different FC profile to that of MoM. FCs for the 28-mm and 36-mm- $\varnothing$ CoC started from 0.1 and 0.09 , respectively, and extended to 0.14 and 0.12 , respectively, at the tenth test repetition. The final FC of the 36-mm- $\varnothing$ CoC prosthesis was $16 \%$ lower than that of the $36-\mathrm{mm}-\varnothing$ one. The average FCs of MoM and CoC prosthesis are shown in the Fig. 5 and 6, respectively.

\subsection{Effect of Particle Size on Friction Coefficient}

Fig. 7 illustrates the impact of artificially induced UHWMPE debris on MoP hip joint prosthesis. At the very beginning of the experiment, the added particle helped reduce FC; FC was slightly less (3\%) than that of the prosthesis interface 'without debris'. The difference 
reduced to zero at $5^{\text {th }}$ experiment. However, FC profiles of prostheses with added particles started to exceed those of the prostheses with no-added particle from the $6^{\text {th }}$ experiment, reaching a difference of $6.5 \%$ at the $10^{\text {th }}$ replication. The post-experiment image of the prosthesis cup (Fig. 3) revealed a layer of deposited PE particles, which confirmed the presence of particles at the interface during the experiment. A large number of particles were also found to attach to the prosthesis head.

\subsection{Effect of Dimples on Metal on Polyethylene Prosthesis Friction Coefficient}

Prosthesis heads with square, circular and triangular dimple arrays were tested against polyethylene acetabulum to determine the resulting FCs (Fig. 8). FCs of all the dimpled prostheses were higher than those of the non-dimpled pairs. For example, the FCs of square and circular arrays were 0.13 at the beginning of the experiment, $13 \%$ higher than that of the nondimple prosthesis. Moreover, the difference increased to $17 \%$ at the end of $10^{\text {th }}$ experiment. On the other hand, prostheses with triangular array started with similar FC values as those of nondimple prostheses, but their FCs gradually increased to $20 \%$ at the end of the $10^{\text {th }}$ test, compared to the non-dimpled MoM. Ito et $\mathrm{al}^{25}$ reported improved frictional coefficient by using similar pattern of dimple. However, our study revealed that large and deep micro dimples, regardless of their arrangements, did not decrease FC, but instead, increase FCs compared to non-dimpled MoP prostheses. From the computation simulation, it has been revealed that an elastic deformation occurred to UHWME, as a consequence, the edge of the deformed UHWME penetrated into the dimple. The depth of the penetration were $8.2 \mu \mathrm{m}$ and $3.2 \mu \mathrm{m}$ under contact pressure $24 \mathrm{MPa}$ and $10 \mathrm{MPa}$, respectively (Fig. 9). Off Couse, the deformation happened due to 
the lower modulus of elasticity of the UHWME compare to that of CrCoMo. The penetrated edge of UHWME are responsible for the increased FC.

\subsection{Effect of Dimples on Metal on Metal Prosthesis Friction Coefficient}

Fig. 10 presents FC trends of dimpled MoM prostheses. FC of MoM prosthesis with square arrays started from a much higher magnitude of 0.17 for the first test, compared to that of non-dimpled prosthesis (0.13), but gradually decreased with increasing number of repeated tests. However, the FC profile of MoM prostheses with non-dimple arrays increased slightly, with an increasing number of repeated tests. The FC of MoM prosthesis with square array reduced by 22\% compared with non-dimpled Isodur-Aesculap, and by 54\%, compared with non-dimpled Metasul-Zimmer MoM hip prosthesis.

The frictional profile for the MoM prosthesis with triangular dimple array was very similar to the one for the MoM prosthesis with squared dimpled array, although its magnitude was slightly higher. While FCs for MoM implants with square and triangular arrays improved with increasing number of tests, the FC for MoM implant with circular array increased substantially, reaching almost $40 \%$ higher than the non-dimpled sodur-Aesculap and MetasulZimmer hip prostheses after the $5^{\text {th }}$ test repetition. It is to be noted that the dimples for the circular array had larger depth and diameter than those for the square and triangular arrays (Table2) because of the manufacturing difficulties. The velocity profiles also confirmed that the squared dimple array had a viscous damping effect, an indication of better lubrication formation

over the contact zone ${ }^{35}$. Therefore, we performed a preliminary in-situ observation of square array in order to observe the lubrication film behavior. To do this, the metal cup was replaced with a glass-made cup. The applied load was reduced in magnitude (500 N), but other 
experimental conditions remained unchanged, including temperature, lubricant and frequency. From the in-situ observation, a clear enhanced film formation was identified, which was believed to be the main reason for lowered friction coefficients of dimpled prosthesis. The enhanced film formation (70nm) is illustrated in Fig. 11.

\section{Discussion}

Friction coefficient, wear rate and film thickness are the three main fundamental aspects of Biotribology $y^{31,39}$. Interface properties and prosthesis geometry are the key areas that can influence the lubrication mechanism of artificial hip joints and, consequently, fluctuation in friction coefficient and wear rate ${ }^{9,32}$. In this study, an investigation was conducted on the frictional behavior of different implant material combinations, such as MoM, MoP, CoC and CoP hip joints prostheses, which are manufactured by two well-renowned medical devicemanufacturing companies-Zimmer and B. Braun. Hence, the clearance and surface roughness were well defined and consistent. Other experimental conditions, such as load (2.5 KN), temperature $\left(37^{\circ} \mathrm{C}\right)$, swing angle $32^{0}$ degree and $25 \%$ bovine serum, were accurately replicated to the clinical conditions of implanted hip joints ${ }^{13,31,40}$.

FC of the 28-mm- $\varnothing$ CoP prosthesis was significantly higher than that of the $36-\mathrm{mm}-\varnothing$ for every test repetition. FC for both CoP head sizes increased with increasing number of test repetitions. The FC trend was similar to those of the MoP prostheses. Interestingly, the FC of CoC for the similar geometrical combinations (28-mm- $\varnothing$ \& 36-mm- $\varnothing)$ are similar trend as per as CoP, however, the scenario changed — there is no significance FC difference between 28-mm- $\varnothing$ \& 36-mm- $\varnothing$ combinations. Clearances of the 36-mm Ø and 28-mm Ø CoC prostheses were 562 $\mu \mathrm{m}$ and $363 \mu \mathrm{m}$ respectively (difference: $199 \mu \mathrm{m}$ ); those of the 36-mm $\varnothing$ and 28-mm $\varnothing \mathrm{MoM}$ 
prostheses were $609 \mu \mathrm{m}$ and $274 \mu \mathrm{m}$ (difference $335 \mu \mathrm{m}$ ), respectively. Therefore, the difference of Clearances of the prostheses is the main factors that influence their frictional behaviors. Using a simplified film thickness equation, Dowson ${ }^{13}$ showed that, when diameter is larger and clearance are minimum the maximum film thickness yield. According to their derived theory, the elastohydrodynamic film thickness $\left(h_{\min }\right)$ between perfectly smooth surfaces is directly proportional to (diameter, $\mathrm{mm})^{2.19}$ and inversely proportional to (clearance, $\left.\mu \mathrm{m}\right)^{0.77}$. Using this equation, the calculated ratio (diameter ${ }^{2.19} /$ clearance $^{0.77}$ ) were 18.37 for the $36-\mathrm{mm}-\varnothing$ MoM hip prosthesis, 33.98 for the 28-mm- $\varnothing$ MoM, 19.54 for the 36-mm- $\varnothing$ CoC and 15.78 for the 28-mm$\varnothing$ CoC. These values show that, despite its larger diameter, the 36-mm- $\varnothing$ MoM cannot improve the prosthesis frictional behaviour unless they have a justified clearance. Similar mechanisms happen to the present experiment, the CoP hip prosthesis as well and larger diameter CoP had lowered FC values. Larger-diameter hip prostheses are reported to have lower risks of dislocation ${ }^{12-14}$. This study reveals that a larger-diameter hip prosthesis, with a minimum diametrical clearance, can improve frictional behaviour.

It is reported that third-body abrasive wear ${ }^{16,17}$, which usually involves fine debris generated from the rubbing area, have lower influence in friction and wear; third body abrasive wear rate is ten times slower than second body abrasive wear ${ }^{9,32}$. However, the slow progression of third body abrasive can decrease the surface fatigue strength which could lead to implant failure over time $^{32}$. In this study, we did not find any significant impact of polyethylene debris on frictional behaviour of MoP prosthesis. Although there was clear evidence of debris at the interface, these could have deformed elastically or plastically due to the high contact pressure, thereby reducing their impact upon wear. At the $6^{\text {th }}$ repeated experiment, there were small differences between the FC of hip prostheses with 'no particle' and those with 'added particles' and the differences 
gradually increased with repeated number of tests. This could be because of an increased number of particles produced at the interface with time.

Fabricated micro dimples are one of the advanced surface modifications for enhancing lubrication, controlling contact pressure and wear debris ${ }^{17,33}$. In this study, micro dimples were found to adversely affect the resultant frictional behaviour of MoP prosthesis, which contradicts findings in the current literature ${ }^{17,23}$. Ito et $\mathrm{al}^{25}$ stated that a concave pattern dimples with $0.5 \mathrm{~mm}$ diameter, $1.2 \mathrm{~mm}$ pitch, and $0.1 \mathrm{~mm}$ depth fabricated on the Co-Cr alloy head could significantly reduce friction and wear rate when rubbing against UHMWPE. Ito and co-investigators' findings ${ }^{25}$ indicate that concave dimpled MoP could be a good solution for preventing excessive UHMWPE wear rate, however, they also suggested that further testing on the dimpled profile should be conducted, using a standard hip simulator. Sawano et al ${ }^{16}$ suggested not to use micro dimples deeper than $1 \mu \mathrm{m}$ since these were found to result in higher friction and wear, however, they used pin-on-disc specimens, which was not a true replication of artificial hip joints in the terms of geometry and clearance. Although our experiments lasted for only 30 minutes, our results showed evidence that the edge of the deformed UHWME penetrate onto the micro dimple of CrCo head were mainly responsible for the increased FC. The numerical analysis (Fig. 9) revealed that height of the UHWMPE deformed edge could be around ' $4 \mu \mathrm{m}$ at the $10 \mathrm{MPa}$ contact pressure' and ' $8 \mu \mathrm{m}$ at the $24 \mathrm{MPa}$ contact pressure' into the dimple profile. These contact edges could act as an interlock and increase the FC.

The most important finding of this study was the effect of micro dimples on MoM prostheses. A significantly lower and steady FC profiles were obtained for micro dimpled MoM prostheses, compared to those of non-dimpled MoM prostheses. A clear evidence of enhanced 
film formation was found, however, we did not measure the quantitative values of film thickness at this stage. The evaluation of film thickness during various sliding and rolling conditions are currently being investigated. The stable and reduced FC of the square and triangular dimple arrays could have a significant impact on the long-lasting stabilization of the hip joint. The circular dimple array, however, resulted in an increased FC. This could have been due to the larger diameter and depth of the circular array, which could have reduced lubrication distribution and film formation. An in situ observation would help understand the lubrication distribution and their associated film formation; this is currently under investigation by our investigational team. The square array showed better performance than the triangular array because of the consistent line-up to the direction of rolling of the prosthesis head. It is to be noted that the rolling of the pendulum hip joint is a unidirectional swing, which is slightly different from the physiological movement of the natural hip joint, but very similar to that of the knee joint. Therefore, the simulator needs to be adapted to simulate the multidirectional movement of the hip joint to obtain an optimum dimple arrangement for hip joints in clinical usages.

There is a lack of investigations on conformal contact of hip joint FC. Most of the published studies report FC values, based on pin- or ball-on-disk (non-conformal) with either unidirectional or reciprocating motion ${ }^{15,41}$. Thus there has been a wide variations in reported FC values $(0.03-$ $1)^{41}$. Scholes et al. ${ }^{15}$ investigated on the frictional behaviour of commercially-available total hip joint prosthesis, however, they provided frictional factor values, which was similar in magnitude to FC values, but varied with the pressure distribution over the head. Moreover, they concentrated only on 28-mm diameter prosthesis, without evaluating the clearance. Dowson et al. ${ }^{13}$ confirmed that prosthesis clearance and size had huge influence on wear mechanism, 
however, they did not provide their friction coefficient outcomes. This study has explored FC of the two most clinically-utilized sizes and material pairs of hip prostheses, along with their respective defined clearance ${ }^{14,39}$. Furthermore, this study has included advanced and well-defined micro dimpled surfaces that resulted in substantial reduced and stable FC. Results of this study could therefore be beneficial to clinicians and prosthesis manufacturing technologists.

\section{Conclusions}

This study consisted of three major parts: a) investigating frictional behavior of two sizes and four material combinations of commercially available hip joint prostheses, b) the influences of artificially added UHWMPE particles at the MoP interface and c) the fabrication of three dimple arrays on spherical hip prosthesis heads and their tribological impact. The conclusion of the student can be pointed out as follows:

- Size and clearance influence the tribological behavior of hip joint prosthesis. A 36-mm- $\varnothing$ CoP, with a clearance of $453 \mu \mathrm{m}$, yielded a significant reduction in FC of 22\%, compared to a 28-mm-Ø CoP. However, the 36-mm- $\varnothing$ MoP and 28-mm- $\varnothing$ MoP hip joint prostheses had similar FC values because of their respective clearance values. FCs of the MoM prostheses had similar trends, but were $12 \%$ lower for the $360 \mathrm{CoC}$ (clearance $562 \mu \mathrm{m}$ ), compared to the 28-mm- $\varnothing \mathrm{CoC}$ (clearance $363 \mu \mathrm{m}$ ). The predicted film thickness and experimental viscous signs supported the friction coefficient results.

- The artificially added UHMPE particles had a minimal effect on the frictional behavior of the 28-mm-Ø Metal on Polyethylene prostheses. The softer UHMWPE particles were found to be deformed under the contact pressure. Post-experiment images revealed the presence of UHMWPE particles at the cup and head interface. 
- The 28-mm-Ø MoP prostheses with three micro dimple arrays yielded a significant increase in FCs, compared to that of the non-dimpled MoP. The edges of 'elastic-deformed UHMWPE cup into rigid micro dimpled prosthesis head' were thought to be the cause of the increased FC. Dimples with smaller diameter and depth are believed to produce lower FCs.

- The square dimple arrayed MoM prosthesis $(28 \mathrm{~mm} \varnothing)$ yielded a significant friction reduction of $24 \%$ and $35 \%$, compared to that of non-dimpled Brown and Zimmer MoM prostheses, respectively. These corresponding percent reductions for the 28-mm-Ø MoM prosthesis with triangular dimple arrays were $19 \%$ and $30 \%$, respectively.

\section{Acknowledgement}

This work is supported by the Excellent Young Researcher project (CZ.1.07/2.3.00/30.0039) from Brno University of Technology, the project "CEITEC - Central European Institute of Technology” (CZ.1.05/1.1.00/02.0068) financed by European Regional Development Fund.

\section{References}

1. Kurtz S, Ong K, Lau E, Mowat F, Halpern M. Projections of primary and revision hip and knee arthroplasty in the United States from 2005 to 2030. The Journal of Bone \& Joint Surgery 2007;89(4):780-785.

2. Lysaght MJ, O'Loughlin JA. Demographic scope and economic magnitude of contemporary organ replacement therapies. ASAIO journal 2000;46(5):515-521.

3. Canadian Joint Replacement Registry 2014 Annual Report. Canadian institute for health information. Canada2014.

4. $\quad$ NJR 10th Annual Report 2013, National Joint Registry UK 2013.

5. Rahman HSA, Choudhury D, Osman NAA, Shasmin HN, Abas W. In vivo and in vitro outcomes of alumina, zirconia and their composited ceramic-on-ceramic hip joints. Journal of the Ceramic Society of Japan 2013;121(4):382-387.

6. Ghosh S, Choudhury D, Das NS, Pingguan - Murphy B. Tribological role of synovial fluid compositions on artificial joints-a systematic review of the last 10 years. Lubrication Science 2014.

7. Nine MJ, Choudhury D, Hee AC, Mootanah R, Osman NAA. Wear Debris Characterization and Corresponding Biological Response: Artificial Hip and Knee Joints. Materials 2014;7(2):980-1016. 
8. Jämsen E, Kouri VP, Olkkonen J, Cör A, Goodman SB, Konttinen YT, Pajarinen J. Characterization of macrophage polarizing cytokines in the aseptic loosening of total hip replacements. Journal of Orthopaedic Research 2014.

9. Rabinowicz E. Friction and wear of materials. 1965.

10. Choudhury D, Ching HA, Mamat AB, Cizek J, Osman NAA, Vrbka M, Hartl M, Krupka I. Fabrication and characterization of DLC coated micro dimples on hip prosthesis heads. Journal of Biomedical Materials Research: Part B - Applied Biomaterials 2014; In press.

11. Vrbka M, Nečas D, Hartl M, Křupka I, Urban F, Gallo J. Visualization of lubricating films between artificial head and cup with respect to real geometry. Biotribology 2015.

12. Gandhe A, Grover M. (i) Head size, does it matter? Current Orthopaedics 2008;22(3):155-164.

13. Dowson D, Hardaker C, Flett M, Isaac GH. A hip joint simulator study of the performance of metal-on-metal joints: Part II: design. The Journal of arthroplasty 2004;19(8):124-130.

14. Ganapathi M. 2014 13/08/2014. Does size of the ball matter in hip replacement? $<$ http://www.ganapathihipkneesurgeon.com/does\%20size\%20of\%20the\%20ball\%20matter\%20i n\%20hip\%20replacement.html>. Accessed 2014 13/08/2014.

15. Scholes S, Unsworth A, Hall R, Scott R. The effects of material combination and lubricant on the friction of total hip prostheses. Wear 2000;241(2):209-213.

16. Sawano $\mathrm{H}$, Warisawa $\mathrm{Si}$, Ishihara S. Study on long life of artificial joints by investigating optimal sliding surface geometry for improvement in wear resistance. Precision engineering 2009;33(4):492-498.

17. Chyr A, Qiu M, Speltz JW, Jacobsen RL, Sanders AP, Raeymaekers B. A patterned microtexture to reduce friction and increase longevity of prosthetic hip joints. Wear 2014;315(1):51-57.

18. Costa $\mathrm{H}$, Hutchings I. Hydrodynamic lubrication of textured steel surfaces under reciprocating sliding conditions. Tribology International 2007;40(8):1227-1238.

19. Sampedro J, Ferre R, Fernández E, Pérez I, Cárcel B, Molina T, Ramos J. Surface Functionalization of AISI 316 Steel by Laser Texturing of Shaped Microcavities with Picosecond Pulses. Physics Procedia 2012;39:636-641.

20. Ali F, Křupka I, Hartl M. Enhancing the parameters of starved EHL point conjunctions by artificially induced replenishment. Tribology International 2013;66:134-142.

21. Křupka I, Hartl M. The effect of surface texturing on thin EHD lubrication films. Tribology International 2007;40(7):1100-1110.

22. Roy $T$, Choudhury D, Bin Mamat A, Pingguan-Murphy B. Fabrication and characterization of micro-dimple array on $\mathrm{A}<<$ sub $>2</$ sub $>\mathrm{O}<$ sub $>3</$ sub $>$ surfaces by using a micro-tooling. Ceramics International 2014;40(1):2381-2388.

23. Qiu M, Chyr A, Sanders AP, Raeymaekers B. Designing prosthetic knee joints with bio-inspired bearing surfaces. Tribology International 2014;77:106-110.

24. Choudhury D, Roy T, Krupka I, Hartl M, Mootanah R. Tribological investigation of ultra-high molecular weight polyethylene against advanced ceramic surfaces in total hip joint replacement. Proceedings of the Institution of Mechanical Engineers, Part J: Journal of Engineering Tribology 2014:1350650114541106.

25. Ito H, Kaneda K, Yuhta T, Nishimura I, Yasuda K, Matsuno T. Reduction of polyethylene wear by concave dimples on the frictional surface in artificial hip joints. The Journal of arthroplasty 2000;15(3):332-338.

26. Zhou X, Galvin AL, Jin Z, Yan X, Fisher J. The influence of concave dimples on the metallic counterface on the wear of ultra-high molecular weight polyethylene. Proceedings of the Institution of Mechanical Engineers, Part J: Journal of Engineering Tribology 2012;226(6):455462. 
27. Choudhury D, Walker R, Roy T, Paul S, Mootanah R. Performance of honed surface profiles to artificial hip joints: An experimental investigation. International Journal of Precision Engineering and Manufacturing 2013;14(10):1847-1853.

28. Choudhury D, Walker R, Shirvani A, Mootanah R, Chelmsford U. The Influence of Honed Surfaces on Metal-on-Metal Hip Joints. Tribology Online 2013;8(3):195-202.

29. Gao L, Meng Q, Liu F, Fisher J, Jin Z. The effect of aspherical geometry and surface texturing on the elastohydrodynamic lubrication of metal-on-metal hip prostheses under physiological loading and motions. Proceedings of the Institution of Mechanical Engineers, Part C: Journal of Mechanical Engineering Science 2010;224(12):2627-2636.

30. Gao L, Yang P, Dymond I, Fisher J, Jin Z. Effect of surface texturing on the elastohydrodynamic lubrication analysis of metal-on-metal hip implants. Tribology International 2010;43(10):18511860.

31. Jin Z, Stone M, Ingham E, Fisher J. (v) Biotribology. Current Orthopaedics 2006;20(1):32-40.

32. Stachowiak G, Batchelor AW. Engineering tribology. Butterworth-Heinemann; 2013.

33. Oshima, Y., Nakano, A., Tsuboi, R., Sasaki, S., (2012). "CFD analysis of real-textured surface processed by laser," Proceedings of 39th Leeds-Lyon Symposium, (CD ROM).

34. U.S.Patent6,045,581. Implantable prosthesis having textured bearing surfaces. USA2010.

35. Choudhury D, Urban F, Vrbka M, Hartl M, Krupka I. A novel tribological study on DLC-coated micro-dimpled orthopedics implant interface. J Mech Behav Biomed Mater 2015;45:121-31.

36. Choudhury D, Ay Ching $H$, Mamat AB, Cizek J, Abu Osman NA, Vrbka M, Hartl M, Krupka I. Fabrication and characterization of DLC coated microdimples on hip prosthesis heads. J Biomed Mater Res B Appl Biomater 2015;103(5):1002-12.

37. Meng $Q$, Liu F, Fisher J, Jin Z. Contact mechanics and lubrication analyses of ceramic-on-metal total hip replacements. Tribology International 2013;63:51-60.

38. Crisco J, Blume J, Teeple E, Fleming B, Jay G. Assuming exponential decay by incorporating viscous damping improves the prediction of the coeffcient of friction in pendulum tests of whole articular joints. Proceedings of the Institution of Mechanical Engineers, Part H: Journal of Engineering in Medicine 2007;221(3):325-333.

39. Dowson D. Bio-tribology. Faraday discussions 2012;156:9-30.

40. Dowson D. Tribological principles in metal-on-metal hip joint design. Proceedings of the Institution of Mechanical Engineers, Part H: Journal of Engineering in Medicine 2006;220(2):161171.

41. Mischler S, Munoz Al. Wear of CoCrMo alloys used in metal-on-metal hip joints: A tribocorrosion appraisal. Wear 2013;297(1):1081-1094.

\section{List of Figures:}

Fig1. Fabricated dimple arrays before polishing: a) square b) triangular c) circular; and after polishing: d) square e) triangular f) circular

Fig 2. Surface roughness profile of dimple before and after the polishing

Fig 3. Pendulum hip simulator 
Fig 4. Effect of size and clearance on friction coefficient in Metal on Polyethylene and Ceramic on Polyethylene hip joints

Fig 5. Effect of size on Friction coefficient in Metal on Metal hip joints

Fig 6. Effect of size and clearance on Friction coefficient in Ceramic on Ceramic hip joints (Biolox ${ }^{\circledR}$ delta, Zimmer)

Fig 7. Effect of polyethylene particles on Friction coefficient in in metal on polyethylene hip joints

Fig 8. Effect of dimples on Friction coefficient in Metal on Polyethylene hip joints

Fig 9. The deformation of Polyethylene into the dimple under pressure of a) $10 \mathrm{MPa}$ and b) 20 $\mathrm{MPa}$

Fig 10. Effect of dimple on Friction coefficient in Metal on Metal hip joints

Fig 11.Effect of square dimple on lubrication (bovine serum) film formation in metal on glass (cup) hip joints

\section{List of Tables:}

Table 1: The geometrical descriptions of Prosthesis head and cup

Table 2. The specification of fabricated dimple arrays

Table 3. Experiment parameters for the friction test (Pendulum hip simulator) 